\title{
ERYTHROPOIETIN PROMOTES NETWORK FORMATION OF TRANSPLANTED ADIPOSE TISSUE-DERIVED MICROVASCULAR FRAGMENTS
}

\author{
P. Karschnia, C. Scheuer, A. Heß, T. Später, M.D. Menger and M.W. Laschke* \\ Institute for Clinical and Experimental Surgery, Saarland University, 66421 Homburg/Saar, Germany
}

\begin{abstract}
The seeding of tissue constructs with adipose tissue-derived microvascular fragments (ad-MVF) is an emerging pre-vascularisation strategy. Ad-MVF rapidly reassemble into new microvascular networks after in vivo implantation. Herein it was analysed whether this process was improved by erythropoietin (EPO). Ad-MVF were isolated from green fluorescent protein $(\mathrm{GFP})^{+}$as well as wild-type C57BL/6 mice and cultivated for $24 \mathrm{~h}$ in medium supplemented with EPO (20 IU/mL) or vehicle. Freshly isolated, non-cultivated ad-MVF served as controls. Protein expression, cell viability and proliferation of ad-MVF were assessed by proteome profiler array and fluorescence microscopy. $\mathrm{GFP}^{+}$ad-MVF were seeded on collagen-glycosaminoglycan matrices, which were implanted into dorsal skinfold chambers of C57BL/6 mice, to analyse their vascularisation over $14 \mathrm{~d}$ by intravital fluorescence microscopy, histology and immunohistochemistry. Cultivation up-regulated the expression of pro- and anti-angiogenic factors within both vehicle- and EPO-treated ad-MVF when compared with non-cultivated controls. Moreover, EPO treatment suppressed cultivation-associated apoptosis and significantly increased the number of proliferating endothelial cells in ad-MVF when compared with vehicletreated and non-cultivated ad-MVF. Accordingly, implanted matrices seeded with EPO-treated ad-MVF exhibited an improved vascularisation, as indicated by a significantly higher functional microvessel density. The matrices of the three groups contained a comparably large fraction of $\mathrm{GFP}^{+}$microvessels originating from the ad-MVF, whereas the tissue surrounding the matrices seeded with EPO-treated ad-MVF exhibited a significantly increased microvessel density when compared with the other two groups. These findings indicated that EPO represents a promising cytokine to further boost the excellent vascularisation properties of ad-MVF in tissue-engineering applications.
\end{abstract}

Keywords: Tissue-engineering, erythropoietin, microvascular fragments, angiogenesis, vascularisation, scaffold, apoptosis, proliferation.

*Address for correspondence: Matthias W. Laschke, MD, PhD, Institute for Clinical and Experimental Surgery, Saarland University, D-66421 Homburg/Saar, Germany

Telephone number: +4968411626554 Fax number: +4968411626553 Email: matthias.laschke@uks.eu

Copyright policy: This article is distributed in accordance with Creative Commons Attribution Licence (http://creativecommons.org/licenses/by-sa/4.0/).

\section{Introduction}

The rapid establishment of a sufficient vascularisation is crucial for the in vivo survival and long-term function of tissue-engineered constructs (Laschke $e t$ al., 2006; Novosel et al., 2011). Since the ingrowth of blood vessels from the surrounding host tissue into the implants is a time-consuming angiogenic process, sophisticated pre-vascularisation strategies have recently been developed (Muscari et al., 2014; Laschke and Menger, 2016a). These strategies focus on the generation of preformed microvascular networks within the constructs, which rapidly interconnect to the host microvasculature after implantation (Laschke et al., 2009).

Adipose tissue-derived microvascular fragments (ad-MVF) represent promising pre-vascularisation units for the seeding of tissue constructs (Laschke and Menger, 2015). They are already used to prevascularise epicardial patches (Shepherd et al., 2007), pancreatic-islets-encapsulating devices (Hiscox et al., 2008), dermal skin substitutes (Frueh et al., 2017a) and scaffolds for bone tissue-engineering (Laschke et al., 2012). Ad-MVF are enzymatically isolated from fat pads and, thus, are a mixture of arterioles, capillaries and venules (Hoying et al., 1996; Frueh et al., 2017b). 
They also contain a large number of mesenchymal stem cells within their physiological niche (McDaniel et al., 2014; Frueh et al., 2017a). Accordingly, ad-MVF exhibit a unique regenerative potential and a high capacity to reassemble in vivo into blood-perfused microvascular networks within implanted tissue constructs (Shepherd et al., 2007; Grässer et al., 2016; Frueh et al., 2017a). However, the latter process still requires several days, resulting in an initial lack of blood supply to the implants (Laschke et al., 2012).

To overcome this problem, freshly isolated adMVF might first be stimulated by a pro-angiogenic compound prior to their seeding on scaffolds, to further improve their initial angiogenic sprouting activity. For this purpose, recombinant human erythropoietin (EPO) represents a promising candidate. EPO is already approved for different clinical indications, such as treatment of anaemia or neuroprotection in preterm infants (Rainville et al., 2016; Fischer et al., 2017). Besides its well-known stimulatory effects on the proliferation and differentiation of red blood cell progenitors (Kimáková et al., 2017), EPO also promotes angiogenesis under pathological conditions (Watanabe et al., 2005) and during tissue regeneration (Calvillo et al., 2003). Moreover, EPO is a potent inhibitor of apoptotic cell death in haematopoietic as well as non-haematopoietic tissues (Socolovsky et al., 2001; Alural et al., 2014) and, thus, might also exert beneficial effects on the viability of ad-MVF.

Based on these considerations, ad-MVF were isolated from epididymal fat pads of donor mice and treated with EPO to assess their viability, proliferation and expression of angiogenesis-related proteins in a controlled in vitro setting. In addition, EPO- and vehicle-treated as well as freshly isolated control adMVF were seeded on collagen-glycosaminoglycan matrices (Integra ${ }^{\circledR}$ ), which were subsequently implanted into dorsal skinfold chambers of recipient mice. The in vivo vascularisation of the implants was studied by means of repetitive intravital fluorescence microscopy, histology and immunohistochemistry.

\section{Materials and Methods}

\section{Animals}

Epididymal fat pads were harvested for the isolation of ad-MVF from C57BL/6 mice and transgenic C57BL/6-TgN(ACTB-EGFP)1Osb/J mice (Institute for Clinical and Experimental Surgery, Saarland University, Homburg/Saar, Germany), with an average age of 11 months and a body weight of $>30 \mathrm{~g}$, to guarantee sufficient amounts of ad-MVF for the scaffold seeding. Dorsal skinfold chambers were implanted in C57BL/6 mice with an average age of 4 months and a body weight of 22-28 g.

The animals were housed one per cage and had access to tap water and standard pellet food (Altromin, Lage, Germany) ad libitum. All animal experiments were approved by the local governmental animal care committee (permission number: 08/2015) and were conducted in accordance with the European legislation on protection of animals and the NIH Guidelines for the Care and Use of Laboratory Animals (NIH Publication \#85-23 Rev. 1985).

\section{Isolation and pre-treatment of ad-MVF}

Donor mice were anaesthetised by intraperitoneal injection of ketamine $(75 \mathrm{mg} / \mathrm{kg}$ body weight; Ursotamin $^{\circledR}$, Serumwerke Bernburg, Bernburg, Germany) and xylazine ( $25 \mathrm{mg} / \mathrm{kg}$ body weight; Rompun ${ }^{\circledR}$, Bayer, Leverkusen, Germany). After midline laparotomy, the animals were killed by incision of the abdominal aorta and the bilateral epididymal fat pads were transferred into Dulbecco's modified Eagle medium (DMEM, supplemented with $10 \%$ foetal calf serum, $100 \mathrm{U} / \mathrm{mL}$ penicillin, $0.1 \mathrm{mg} /$ $\mathrm{mL}$ streptomycin; Biochrom, Berlin, Germany) (Fig. 1a). After careful mechanical dissection using a fine scissor, the fat pads were enzymatically digested for the isolation of ad-MVF, as previously described in detail by Frueh et al. (2017b) (Fig. 1b).

Directly after isolation, ad-MVF were cultivated for $24 \mathrm{~h}$ under humidified conditions $\left(37^{\circ} \mathrm{C}, 5 \% \mathrm{CO}_{2}\right)$ in endothelial cell growth medium MV (ECGM MV; PromoCell, Heidelberg, Germany) supplemented with $20 \mathrm{IU} / \mathrm{mL}$ EPO beta (NeoRecormon ${ }^{\circledR}$, Roche, Basel, Switzerland) or vehicle (saline solution), whereas freshly isolated, non-cultivated ad-MVF served as controls. A short-term cultivation of $24 \mathrm{~h}$ does not affect the typical vessel morphology of ad-MVF, which exhibit a lumen surrounded by an endothelial layer and perivascular cells (Laschke et al., 2015). Moreover, the $24 \mathrm{~h}$ cultivation period was chosen to provide enough time for an EPO-induced up- or down-regulation of protein expression (Wang et al., 2008). The cultivated and non-cultivated adMVF were washed in phosphate-buffered saline (PBS) and further analysed in vitro and in vivo.

\section{Protein expression of ad-MVF}

For protein expression analyses, ad-MVF were harvested and pooled from 21 C57BL/6 donor mice. One third of the ad-MVF was cultivated for $24 \mathrm{~h}$ in medium containing EPO, whereas the second third was incubated in medium supplemented with vehicle. The last third of the ad-MVF was not cultivated and served as freshly isolated control. Subsequently, the expression of angiogenesisrelated proteins was screened using a proteome profiler mouse angiogenesis array kit, according to the manufacturer's instructions (R\&D Systems, Wiesbaden, Germany). Whole protein extracts were generated by homogenising the ad-MVF in lysis buffer [10 mM Tris pH 7.5, $10 \mathrm{mM} \mathrm{NaCl}, 0.1 \mathrm{mM}$ ethylenediaminetetraacetic acid (EDTA), $0.5 \%$ Triton- $X$ $100,0.02 \% \mathrm{NaN}_{3}, 0.2 \mathrm{mM}$ phenylmethylsulphonyl fluoride (PMSF), 1:75 v/v protease inhibitor cocktail and $1: 100 \mathrm{v} / \mathrm{v}$ phosphatase inhibitor cocktail (all from Sigma-Aldrich)]. After $30 \mathrm{~min}$ of incubation 
on ice, the samples were centrifuged at $4{ }^{\circ} \mathrm{C}$ for $30 \mathrm{~min}$ at $16,000 \times g$ and the supernatants were used for whole protein extraction. For the array, $250 \mu \mathrm{g}$ of protein per group was exposed for $1 \mathrm{~h}$ at room temperature to a cocktail of 53 biotinylated detection antibodies against soluble growth and differentiation factors, matrix components, membrane-bound receptors and intracellular signalling molecules. The labelled sample cocktail was incubated at $4{ }^{\circ} \mathrm{C}$ overnight with the capture antibodies-spotted array membrane. The visualisation of the labelled specific target proteins ( $n=2$ technical replicates) was achieved with streptavidin-horseradish peroxidase and chemiluminescent detection reagents using an Intas ECL Chemocam Imager (Intas Science Imaging Instruments $\mathrm{GmbH}$, Göttingen, Germany).

\section{Implant seeding with ad-MVF}

Vehicle- and EPO-treated as well as freshly isolated ad-MVF were seeded onto $3 \mathrm{~mm}$ collagenglycosaminoglycan matrices, which were cut out of a 1.3 mm-thick Integra ${ }^{\circledR}$ Dermal Regeneration Template Single Layer without silicone sheet (Integra $\mathrm{GmbH}$, Ratingen, Germany) using a $3 \mathrm{~mm}$ biopsy punch (Kai Europe GmbH, Solingen, Germany). The matrices were placed onto a $500 \mu \mathrm{m}$ cell strainer (pluriSelect Life Science, Leipzig, Germany) and the ad-MVF, suspended in $10 \mu \mathrm{L}$ PBS, were transferred onto the samples with a $100 \mu \mathrm{L}$ pipette (Eppendorf). Additional negative pressure was induced with a syringe underneath the matrices, to trap the fragments within the central scaffold pores (Frueh et al., 2017a).

\section{Dorsal skinfold chamber model}

For in vivo analyses, ad-MVF were isolated from the epididymal fat pads of 12 C57BL/6-TgN(ACTBEGFP)1Osb/J donor mice. Again, one third of the adMVF was cultivated for $24 \mathrm{~h}$ in medium containing EPO, whereas the second third was incubated in medium supplemented with vehicle. The last third of the ad-MVF was not cultivated and served as freshly isolated control. Then, the ad-MVF were seeded onto 24 collagen-glycosaminoglycan matrices, which were implanted into the dorsal skinfold chambers (IROLA Industriekomponenten $\mathrm{GmbH} \& \mathrm{Co}$. KG, Schonach, Germany) of C57BL/ 6 recipient mice ( $n=8$ per group) (Fig. 1c,d). The chamber preparation was performed as previously described (Laschke et al., 2011). After the surgical procedure, the animals were allowed to recover for $48 \mathrm{~h}$ prior to matrix implantation, to prevent postoperative alterations of the microcirculation.

The ad-MVF-seeded matrices were analysed directly after implantation and on days 3, 6, 10 and 14 by means of intravital fluorescence microscopy. For this purpose, the animals were anaesthetised by intraperitoneal injection of ketamine $(75 \mathrm{mg} / \mathrm{kg}$ body weight; Ursotamin $\left.{ }^{\circledR}\right)$ and xylazine $(25 \mathrm{mg} / \mathrm{kg}$ body weight; Rompun ${ }^{\circledR}$ ). Subsequently, $0.1 \mathrm{~mL}$ of $5 \%$ fluorescein isothiocyanate (FITC)-labelled dextran 150,000 (Sigma-Aldrich) was applied intravenously in the retrobulbary space for intravascular plasma staining. The mice were fixed on an acrylic glass stage and the observation window of the chamber was placed horizontally under a Zeiss Axiotech microscope (Zeiss) with a $100 \mathrm{~W}$ mercury lamp. The tissue was visualised by epi-illumination with blue light and the microscopic images were recorded by a charge-coupled device video camera (FK6990; Pieper, Schwerte, Germany) and transferred to a DVD system (DVD-HR775; Samsung Electronics) for off-line analysis. After the last microscopy on day 14, the mice were euthanised with an overdose of anaesthetics and the dorsal skinfold preparations were carefully excised for histological and immunohistochemical analyses.

\section{Microcirculatory analysis}

Image analysis was performed with the computerassisted off-line analysis system CapImage (Zeintl, Heidelberg, Germany). The vascularisation of the
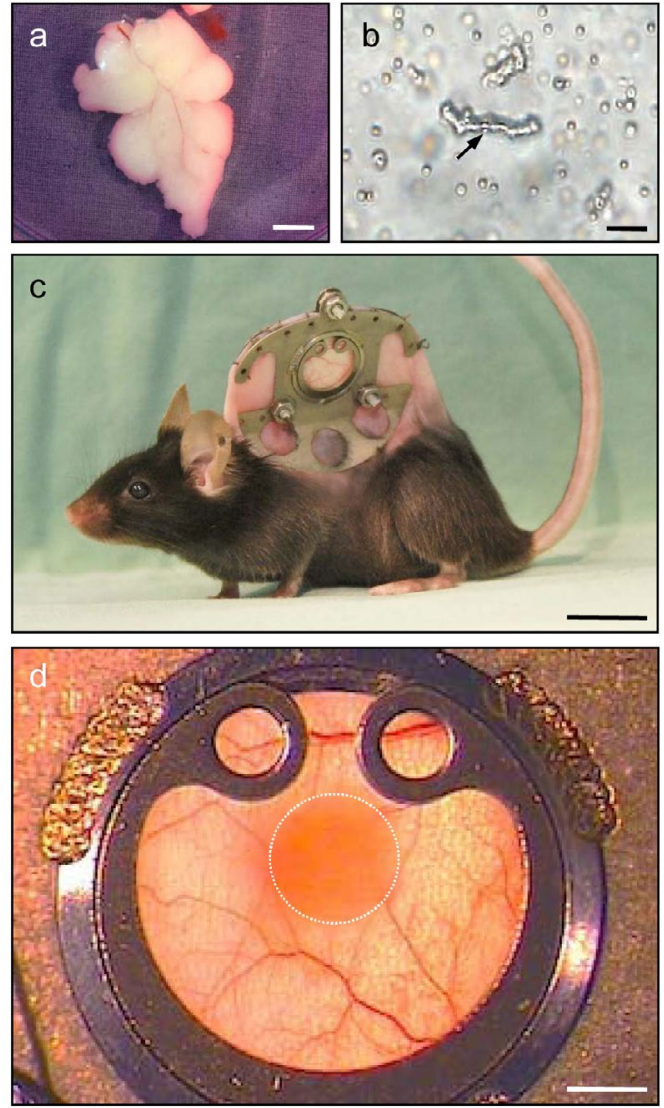

Fig. 1. (a) Excised epididymal fat pad of a donor mouse. Scale bar: 4 mm. (b) Brightfield microscopy of an ad-MVF (arrow) directly after the isolation from the fat pad. Scale bar: $30 \mu \mathrm{m}$. (c) C57BL/6 mouse with a dorsal skinfold chamber. Scale bar: $14 \mathrm{~mm}$. (d) Observation window of the dorsal skinfold chamber after implantation of an adMVF-seeded collagen-glycosaminoglycan matrix (borders marked by dotted line). Scale bar: $2 \mathrm{~mm}$. 
Table 1a. Expression of pro-angiogenic proteins (percentage of freshly isolated control) in vehicleand EPO-treated ad-MVF, as assessed by a proteome profiler mouse angiogenesis array. Data are presented as mean of two technical replicates.

\begin{tabular}{|c|c|c|}
\hline Protein & Expression (pe & isolated control) \\
\hline Pro-angiogenic & Vehicle & EPO \\
\hline MMP-3 & 847.5 & 252.4 \\
\hline PIGF-2 & 637.7 & 584.8 \\
\hline KC/CXCL1/CINC-1/GRO-alpha & 642.2 & 528.2 \\
\hline MMP-8 & 527.9 & 625.0 \\
\hline PDGF-AA & 507.6 & 196.0 \\
\hline IL-1alpha & 381.3 & 262.8 \\
\hline MCP-1/CCL2/JE & 350.2 & 222.2 \\
\hline GM-CSF & 333.0 & 282.7 \\
\hline Endothelin-1/ET-1 & 316.7 & 301.1 \\
\hline IL-10/CSIF & 289.1 & 265.2 \\
\hline Proliferin & 276.8 & 212.0 \\
\hline HB-EGF & 258.4 & 236.0 \\
\hline CXCL 16 & 259.0 & 180.1 \\
\hline MMP-9 & 231.8 & 158.0 \\
\hline MIP-1alpha & 231.8 & 93.6 \\
\hline VEGF B/VRF & 236.0 & 150.1 \\
\hline IGFBP-3 & 225.3 & 254.0 \\
\hline Osteopontin/OPN & 200.4 & 170.6 \\
\hline Angiogenin/ANG & 191.5 & 163.7 \\
\hline Fractalkine/CX3CL 1 & 173.5 & 271.2 \\
\hline IGFBP-1 & 170.7 & 111.3 \\
\hline KGF/FGF-7 & 160.8 & 196.9 \\
\hline PD-ECGF & 164.2 & 139.6 \\
\hline SDF-1/CXCL 12 & 137.5 & 154.7 \\
\hline Angiopoietin-1/Ang-1 & 131.8 & 124.0 \\
\hline Amphiregulin/AR & 130.7 & 103.1 \\
\hline HGF & 131.5 & 122.9 \\
\hline IL-1beta & 128.3 & 145.2 \\
\hline FGF acid/FGF-1/ECGF/HBGF-1 & 123.6 & 116.6 \\
\hline FGF basic/FGF-2 & 128.0 & 116.9 \\
\hline VEGF/VPF & 119.1 & 145.1 \\
\hline Endoglin/CD105 & 111.4 & 88.5 \\
\hline Coagulator Factor III/Tissue Factor/TF & 111.0 & 79.0 \\
\hline IGFBP-2 & 104.1 & 109.2 \\
\hline Leptin/OB & 105.0 & 60.8 \\
\hline EGF & 92.8 & 118.8 \\
\hline DLL4 & 82.3 & 78.4 \\
\hline Cyr61/CCN1, IGFBP-10 & 81.3 & 78.4 \\
\hline NOV/CCN3/IGFBP-9 & 41.5 & 31.8 \\
\hline
\end{tabular}

implants was assessed at a magnification of $\times 460$ in 8 standardised regions of interest (ROIs). Perfused ROIs (in percentage of all ROIs) were defined as areas exhibiting either newly developed red blood cell (RBC)-perfused microvessels or re-perfused green fluorescent protein (GFP) $)^{+}$ad-MVF. Furthermore, the functional microvessel density, i.e. the length of all RBC-perfused microvessels per ROI given in $\mathrm{cm} / \mathrm{cm}^{2}$, was determined. Finally, the diameter $(d$; given in $\mu \mathrm{m})$ and the centreline RBC velocity ( $v$; given in $\mu \mathrm{m} / \mathrm{s}$ ) of 40 randomly selected perfused microvessels within the implants were measured. The wall shear rate $\left(y\right.$; given in $\left.\mathrm{s}^{-1}\right)$ of these vessels was additionally calculated based on the Newtonian definition: $y=8 \times v / d$.

\section{Histology and immunohistochemistry}

Vehicle- and EPO-treated as well as freshly isolated ad-MVF from 6 donor mice were transferred onto 4 collagen-glycosaminoglycan matrices per group, which were directly processed for immunohistochemical analyses of cell viability 
Table 1b. Expression of anti-angiogenic proteins (percentage of freshly isolated control) in vehicle- and EPO-treated ad-MVF, as assessed by a proteome profiler mouse angiogenesis array. Data are presented as mean of two technical replicates.

\begin{tabular}{|l|c|c|}
\hline Protein & \multicolumn{2}{|c|}{ Expression (percentage of freshly isolated control) } \\
\hline Anti-angiogenic & Vehicle & EPO \\
\hline Pentraxin-3/PTX3/TSG-14 & 591.8 & 384.0 \\
\hline TIMP-1 & 272.6 & 241.6 \\
\hline Serpin F1/PEDF & 217.7 & 159.3 \\
\hline Thrombospondin-2/TSP-2 & 220.4 & 141.6 \\
\hline Prolactin/PRL & 216.7 & 155.0 \\
\hline IP-10/CXCL 10 & 180.2 & 123.2 \\
\hline Angiopoietin-3/Ang-3 & 138.4 & 141.0 \\
\hline ADAMTS1/METH1 & 140.0 & 134.0 \\
\hline PDGF-AB/BB & 131.9 & 94.6 \\
\hline Platelet factor 4/CXCL4/PF4 & 128.4 & 94.7 \\
\hline Serpin E1/PAI-1 & 126.1 & 128.6 \\
\hline DPP IV/CD26 & 115.2 & 89.5 \\
\hline Endostatin/Collagen VIII & 111.5 & 75.8 \\
\hline TIMP-4 & 81.9 & 67.6 \\
\hline
\end{tabular}

and proliferation. For this purpose, the specimens were fixed in formalin, embedded in paraffin and cut into $3 \mu \mathrm{m}$-thick sections. To determine immunohistochemically the number of cleaved caspase (Casp) $-3^{+}$apoptotic cells and $\mathrm{Ki}^{+} 7^{+}$ proliferating cells, the sections were co-stained with a rabbit polyclonal anti-Casp-3 antibody (1 : 100; New England Biolabs) or a rabbit polyclonal anti-Ki67 antibody (1: 500; Abcam) and, additionally, with a monoclonal rat-anti-mouse antibody against CD31 (1 : 100; dianova GmbH, Hamburg, Germany) as primary antibodies. A biotinylated goat-anti-rabbit antibody (ready-to-use; Abcam) and a goat-anti-rat Alexa488 antibody (1 : 200; Thermo Fisher Scientific $\mathrm{GmbH}$ ) served as secondary antibodies. The detection of the biotinylated antibody was performed using Alexa555-labelled-streptavidin (1:50; Thermo Fisher Scientific $\mathrm{GmbH})$. To merge the images exactly, cell nuclei on each section were stained with Hoechst 33342 (2 $\mu \mathrm{g} / \mathrm{mL}$; Sigma-Aldrich). Quantitative analyses of the sections included the determination of the fractions of Casp- $3^{+}$and $\mathrm{Ki} 67^{+}$cells (given in percentage) in randomly selected ad-MVF within the matrices, including at least 100 endothelial and perivascular cells per sample. The sections were examined with a BX60 microscope (Olympus).

For the immunohistochemical analysis of sagittal cross-sections of the implanted matrices and their surrounding host tissue, co-staining with a monoclonal rat-anti-mouse antibody against CD31 (1 : 100; dianova $\mathrm{GmbH}$ ), to detect endothelial cells, and with a goat polyclonal anti-GFP antibody (1 : 200; Biomol, Hamburg, Germany), to enhance GFP-fluorescence, was performed. As secondary antibodies, a goat-anti-rat Alexa555 antibody ( 1 : 200; Thermo Fisher Scientific $\mathrm{GmbH}$ ) and a biotinylated donkey-anti-goat antibody, which was detected by
Alexa488-labelled-streptavidin (1 : 50 each; Thermo Fisher Scientific $\mathrm{GmbH}$ ), were used. The sections were placed in Coplin jars (Thermo Fisher Scientific $\mathrm{GmbH}$ ) with $0.05 \%$ citraconic anhydride solution (pH 7.4) for $1 \mathrm{~h}$ at $98^{\circ} \mathrm{C}$ and, subsequently, incubated overnight at $4{ }^{\circ} \mathrm{C}$ with the primary antibody. The labelling with the appropriate secondary antibody was performed at $37^{\circ} \mathrm{C}$ for $1 \mathrm{~h}$. Again, cell nuclei

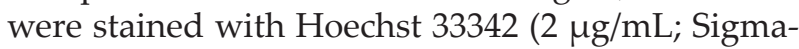
Aldrich). The density of $\mathrm{CD} 31^{+}$microvessels (given in $\mathrm{mm}^{-2}$ ) and the fraction of $\mathrm{GFP}^{+}$microvessels (given in percentage) in the border and centre of the implanted matrices was assessed quantitatively using a BX60 microscope (Olympus).

\section{Statistics}

Data were tested for normal distribution and equal variance. Differences among the groups were analysed by ANOVA followed by the Student-NewmanKeuls post-hoc test. In case of non-parametric data, differences were assessed by ANOVA on Ranks followed by the Dunn's post-hoc test (Prism 7.0a; GraphPad Software Inc.). All values are expressed as mean \pm standard error of the mean (SEM). Statistical significance was accepted for a value of $p<0.05$.

\section{Results}

\section{Protein expression of ad-MVF}

To study the effect of EPO on the angiogenic activity of ad-MVF, a proteome profiler mouse angiogenesis array for the analysis of 53 angiogenesis-related proteins was performed. Cultivation for $24 \mathrm{~h}$ markedly changed the expression profile of ad-MVF when compared with freshly isolated, non-cultivated controls (Table 1a,b). Most of the analysed pro- and 
anti-angiogenic factors presented higher expression levels in vehicle- and EPO-treated ad-MVF. In both groups, a strong up-regulation was detected for the pro-angiogenic factors placental-derived growth factor (PIGF)-2, keratinocyte chemoattractant (KC) and matrix metalloproteinase (MMP)-8 as well as for the anti-angiogenic factor pentraxin-3 (Table $1 \mathbf{a}, \mathbf{b})$. Notably, individual differences were detected between vehicle- and EPO-treated ad-MVF. For instance, EPO-treated ad-MVF exhibited a lower expression of MMP-3, platelet-derived growth factor (PDGF)-AA and pentraxin. However, the difference in the overall protein expression profiles between the two cultivated groups was considerably less pronounced when compared with freshly isolated controls. This indicated a strong cultivation-induced activation of ad-MVF, which was only marginally affected by additional EPO treatment.

\section{Viability and proliferation of ad-MVF}

Ad-MVF exhibit a typical vessel morphology with CD $31^{+}$endothelial cells that are surrounded by stabilising $\mathrm{CD}^{-} 1^{-}$perivascular cells (Laschke et al., 2012). To assess the viability and proliferative activity of these two cell populations in ad-MVF, immunohistochemical stainings of the apoptosis marker Casp-3 and the proliferation marker Ki67 were performed (Fig. 2a-f). These stainings revealed that vehicle-treated ad-MVF contained a significantly higher fraction of apoptotic perivascular cells when compared with freshly isolated, noncultivated controls (Fig. 2a,b,g). This negative effect of cultivation on cell viability was completely prevented by EPO treatment (Fig. 2c,g). Moreover, EPO-treated ad-MVF presented a significantly larger number of $\mathrm{Ki} 67^{+}$endothelial cells when compared with the other two groups (Fig. 2d-f,h).

\section{Vascularisation of implanted ad-MVF-seeded matrices}

To analyse the in vivo vascularisation capacity of vehicle- and EPO-treated as well as freshly isolated ad-MVF, cells were seeded onto collagenglycosaminoglycan matrices, which were implanted into the dorsal skinfold chamber of recipient mice. Intravital fluorescence microscopy allowed for the
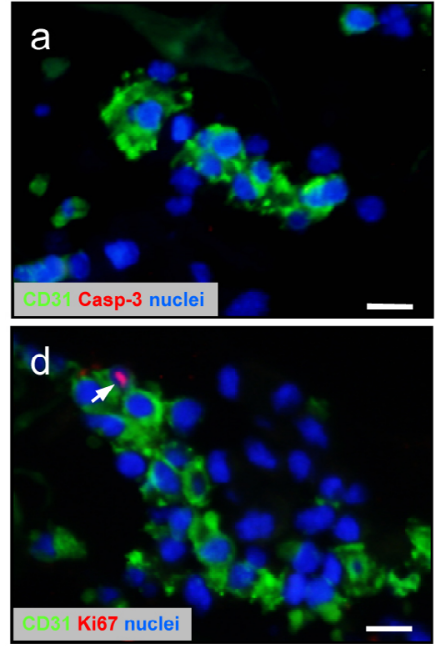

g

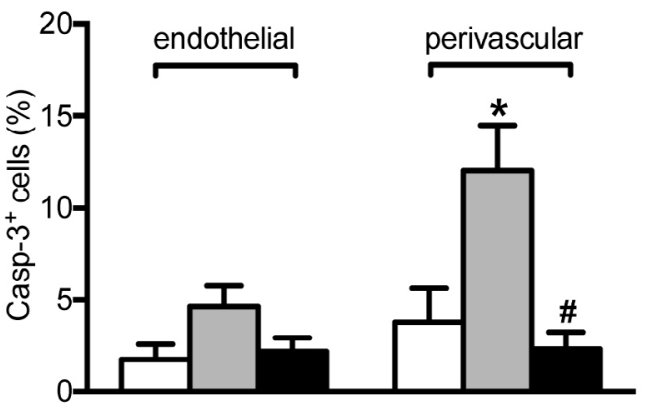

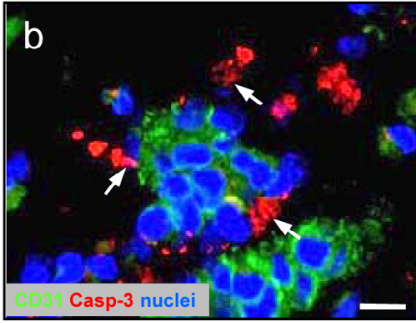
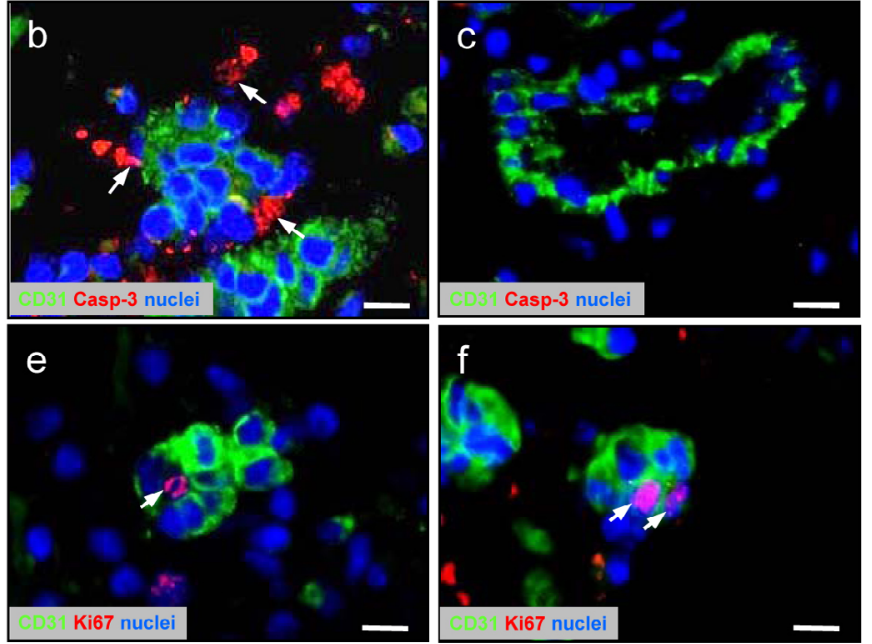

h

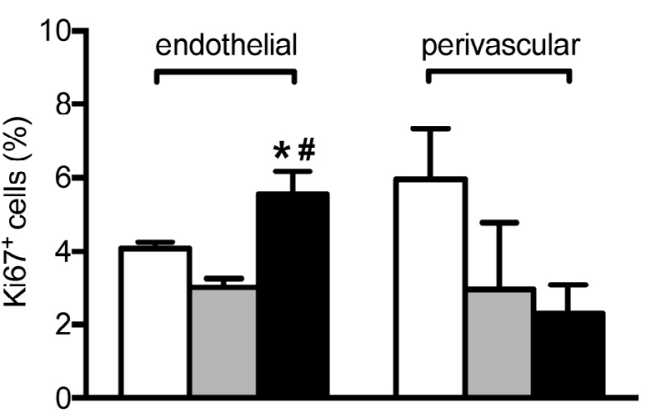

Fig. 2. (a-f) Fluorescence microscopy of (b,e) vehicle-treated, $(\mathbf{c}, \mathbf{f})$ EPO-treated and (a,d) freshly isolated ad-MVF directly after seeding onto collagen-glycosaminoglycan matrices. Staining was performed with Hoechst 33342 (a-f; blue) to identify cell nuclei and anti-CD31 (a-f; green) for the detection of endothelial cells in combination with anti-Casp-3 (a-c; red, arrows) for the detection of apoptotic cells or anti-Ki67 (d-f; red, arrows) for the detection of proliferating cells. Scale bars: $10 \mu \mathrm{m}$. (g,h) Casp-3 $3^{+}$apoptotic (g, $\%$ ) and $\mathrm{Ki}^{+} 7^{+}$proliferating (h, \%) cells within vehicle-treated (grey bars, $n=4$ ), EPO-treated (black bars, $n=4$ ) and freshly isolated ad-MVF (white bars, $n=4)$. ${ }^{*} p<0.05$ vs. freshly isolated ad-MVF. ${ }^{*} p<0.05$ vs.

vehicle-treated ad-MVF. 
repetitive visualisation of functional, blood-perfused microvessels within the implants throughout an observation period of $14 \mathrm{~d}$ (Fig. 3a-o).

Of interest, a few blood-perfused microvessels were already detected in the matrices seeded with EPO-treated and freshly isolated ad-MVF on day 3 , whereas matrices seeded with vehicle-treated ad-MVF did not demonstrate blood perfusion at this early time point after implantation (Fig. 3p,q). During the following time course, the ad-MVF of the three groups developed many interconnections to the blood vessels of the surrounding host tissue, resulting in a comparably high rate of perfused ROIs of $100 \%$ on days 10 and 14 (Fig. 3p). However, more detailed analyses of the newly developing microvascular networks within the implants revealed a significantly higher functional microvessel density between days 6 and 14 in the EPO group (Fig. 3q). Moreover, individual microvessels in these networks presented smaller diameters as well as higher centreline RBC velocities and shear rates when compared with the microvessels in the vehicle group, indicating an accelerated microvascular maturation and remodelling process (Fig. 4a-1).

The final vascularisation of the implanted matrices on day 14 was additionally assessed by means of immunohistochemical analyses. Because the matrices were seeded with $\mathrm{GFP}^{+}$ad-MVF from transgenic C57BL/6-TgN(ACTB-EGFP)1Osb/J mice and implanted into dorsal skinfold chambers of $\mathrm{GFP}^{-}$recipient animals, these analyses enabled not only the assessment of the microvessel density within the implants and their surrounding border zones, but also to differentiate between microvessels originating from seeded $\mathrm{GFP}^{+}$ad-MVF and ingrowing GFPmicrovessels of the host tissue (Fig. 5a-k).

The matrices seeded with vehicle- and EPOtreated as well as freshly isolated ad-MVF exhibited a comparable final density of $\mathrm{CD} 31^{+}$microvessels in their centre zones (Fig. 5l). In contrast, EPO treatment resulted in a significantly increased microvessel density within the surrounding host tissue at the matrices' border zones when compared with the other two groups (Fig. 51). Moreover, the implants

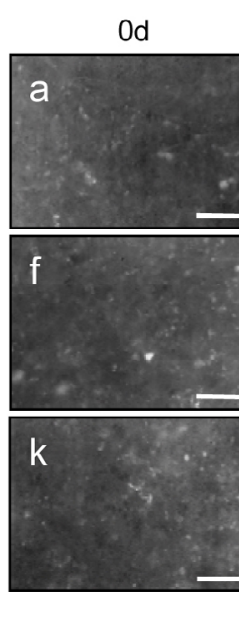

$\mathrm{p}$

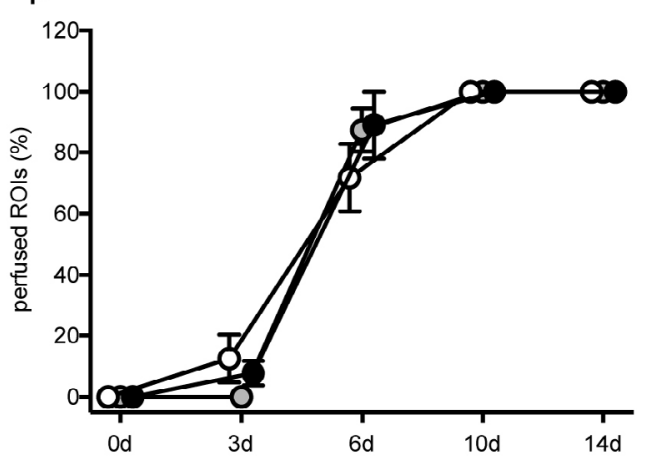

$3 d$
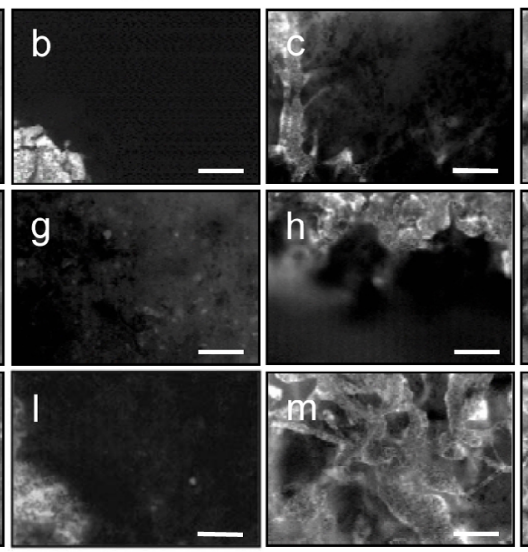

q

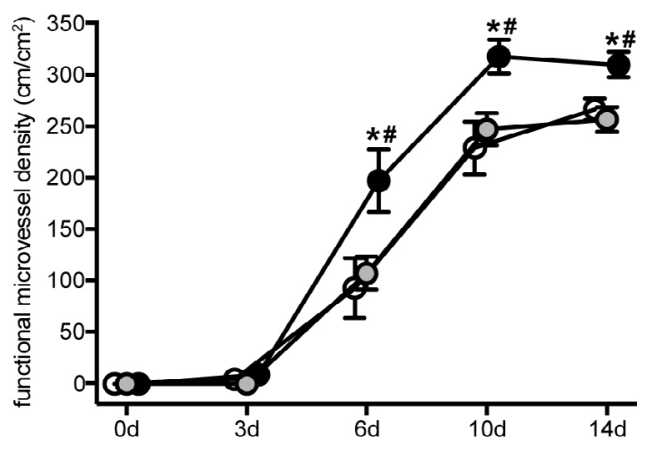

Fig. 3. (a-o) Intravital fluorescence microscopy (blue light epi-illumination with contrast enhancement by $5 \%$ FITC-labelled dextran 150,000, intravenously) of collagen-glycosaminoglycan matrices seeded with $(\mathbf{f}-\mathbf{j})$ vehicle-treated, (k-o) EPO-treated and (a-e) freshly isolated ad-MVF directly (0 d) as well as on days 3, 6, 10 and 14 after implantation into the dorsal skinfold chamber of C57BL/6 mice. Scale bars: $125 \mu \mathrm{m}$. Perfused ROIs ( $\mathbf{p}, \%)$ and functional microvessel density $\left(\mathbf{q}, \mathrm{cm} / \mathrm{cm}^{2}\right)$ of matrices seeded with vehicle-treated (grey circles, $n=8$ ), EPO-treated (black circles, $n=8$ ) and freshly isolated ad-MVF (white circles, $n=8$ ) directly $(0 \mathrm{~d})$ as well as on days 3, 6, 10 and 14 after implantation, as assessed by intravital fluorescence microscopy and computer-assisted image analysis. Means \pm SEM. ${ }^{*} p<0.05 v$ s. freshly isolated ad-MVF. ${ }^{\#} p<0.05$ vs. vehicle-treated ad-MVF. 
of all groups contained a large fraction of $60-70 \%$ $\mathrm{GFP}^{+}$microvessels originating from the seeded adMVF (Fig. 5m). These microvessels even grew out of the implants into the surrounding border zones (Fig. $5 \mathbf{c}-\mathbf{k}, \mathbf{m})$. There was no significant difference in the fraction of $\mathrm{GFP}^{+}$microvessels among the groups.

\section{Discussion}

The present study demonstrated that EPO exerted beneficial effects on isolated ad-MVF. Short-term, $24 \mathrm{~h}$ exposure to this cytokine maintained the viability and increased the proliferation of cultivated ad-MVF. Accordingly, EPO-treated ad-MVF exhibited an improved capacity to reassemble into new, functional microvascular networks after seeding onto collagenglycosaminoglycan matrices and in vivo implantation.

FDA-approved EPO beta was used, which is already applied in many in vitro and in vivo studies. Therefore, dose-finding experiments were not performed, but the used dose of EPO was based on the literature. Watanabe et al. (2005) show that doses of 1-20 IU/mL EPO stimulate the proliferation of bovine endothelial cells, whereas $50 \mathrm{IU} / \mathrm{mL}$ EPO induce adverse effects on their proliferative activity. However, the herein analysed ad-MVF were of mouse origin and did not only consist of endothelial cells. Accordingly, their reactivity against EPO treatment may better correlate with results from an ex situ mouse aortic ring assay, as performed by Nakano et al. (2007). In their assay, $10 \mathrm{IU} / \mathrm{mL}$ EPO only induces a moderate vascular sprouting when compared with vascular endothelial growth factor (VEGF)-stimulated controls, indicating that higher doses of EPO may be more effective. Based on these considerations, a concentration of $20 \mathrm{IU} / \mathrm{mL}$ EPO was used for the $e x$ situ pre-treatment of ad-MVF. By this, the number
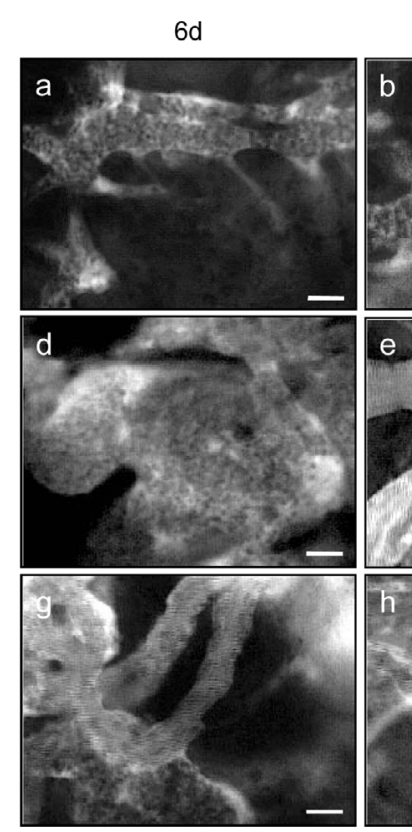

j

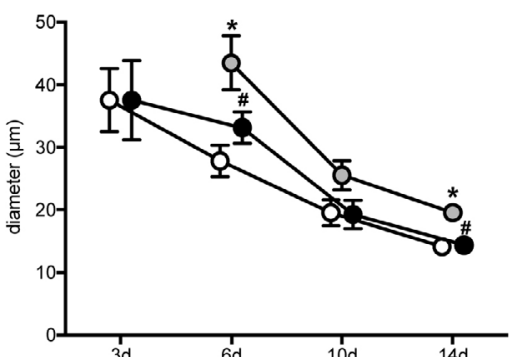

$\mathrm{k}$

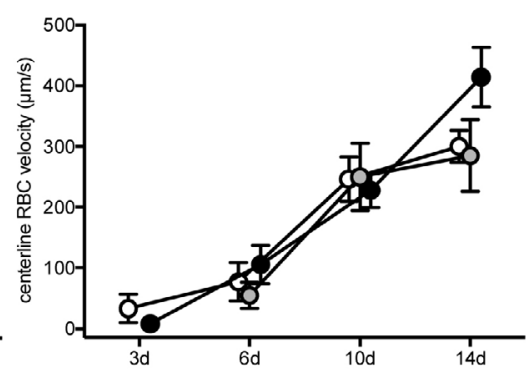

I

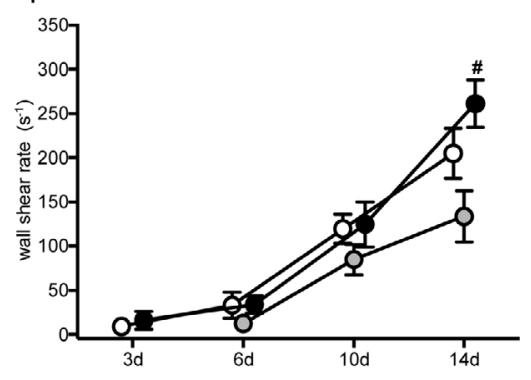

$14 d$

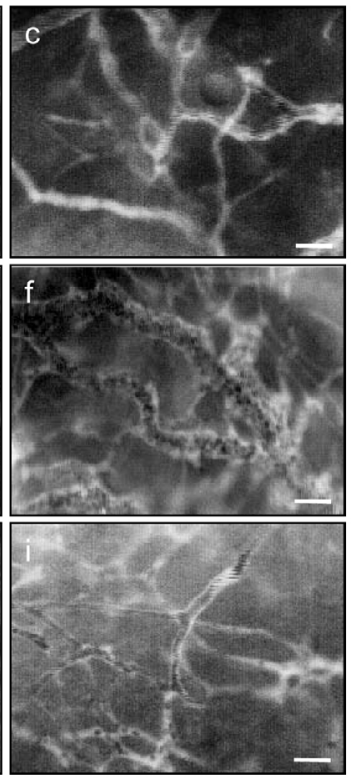

Fig. 4. (a-i) Intravital fluorescence microscopy (blue light epiillumination with contrast enhancement by 5\% FITC-labelled dextran 150,000, intravenously) of collagen-glycosaminoglycan matrices seeded with (d-f) vehicletreated, (g-i) EPO-treated and (a-c) freshly isolated ad-MVF on days 6, 10 and 14 after implantation into the dorsal skinfold chamber of C57BL/ 6 mice. Scale bars: $40 \mu \mathrm{m}$. Diameter $(\mathbf{j}, \mu \mathrm{m})$, centreline RBC velocity $(\mathbf{k}$, $\mu \mathrm{m} / \mathrm{s})$ and wall shear rate $\left(\mathbf{1}, \mathrm{s}^{-1}\right)$ of microvessels within matrices seeded with vehicle-treated (grey circles, $n=8$ ), EPO-treated (black circles, $n=8)$ and freshly isolated ad-MVF (white circles, $n=8$ ) on days 3,6 , 10 and 14 after implantation, as assessed by intravital fluorescence microscopy and computer-assisted image analysis. Means \pm SEM. ${ }^{*} p<0.05$ vs. freshly isolated ad-MVF. ${ }^{\#} p<0.05$ vs. vehicle-treated ad-MVF. 

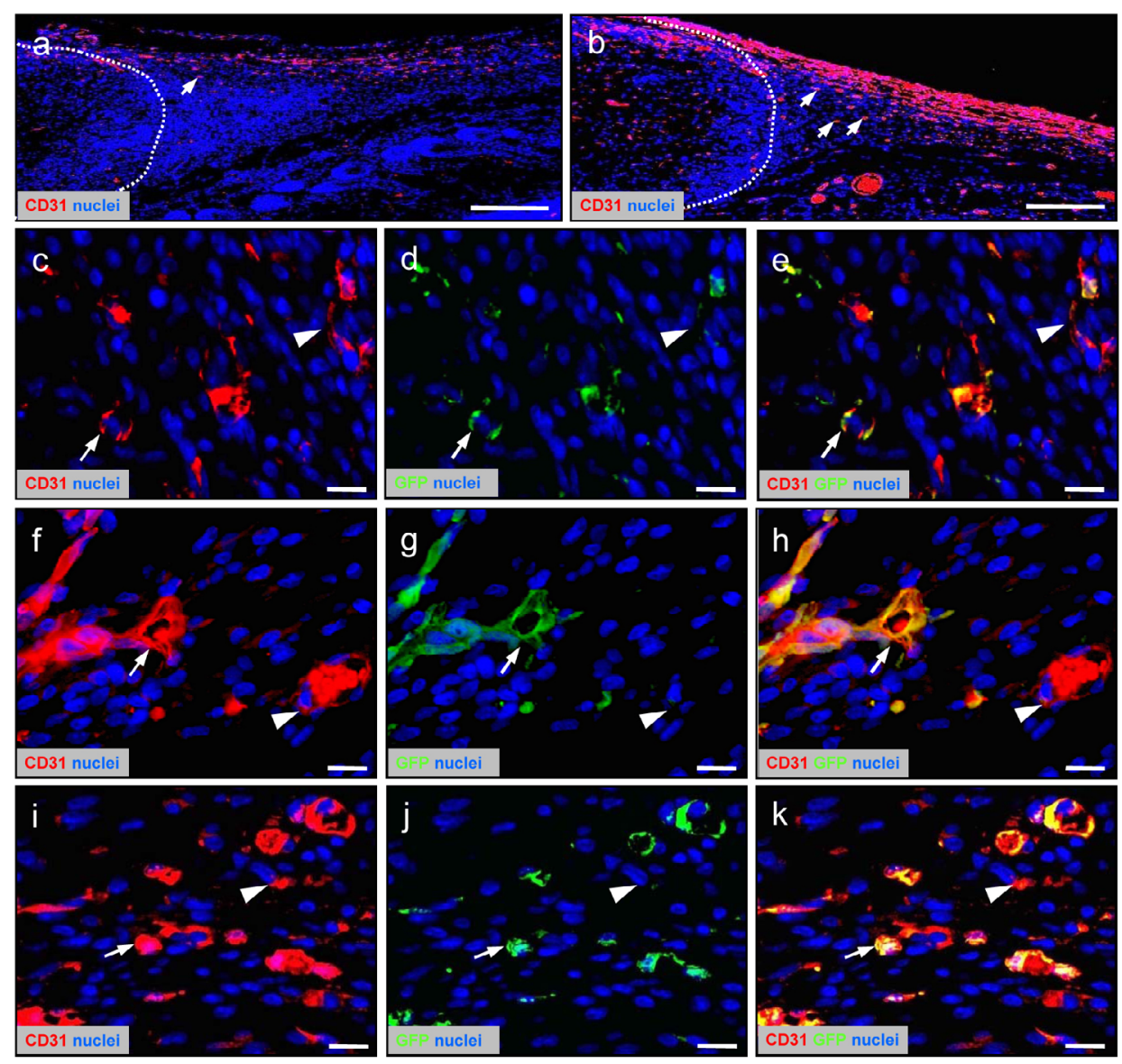

I

$\mathrm{m}$
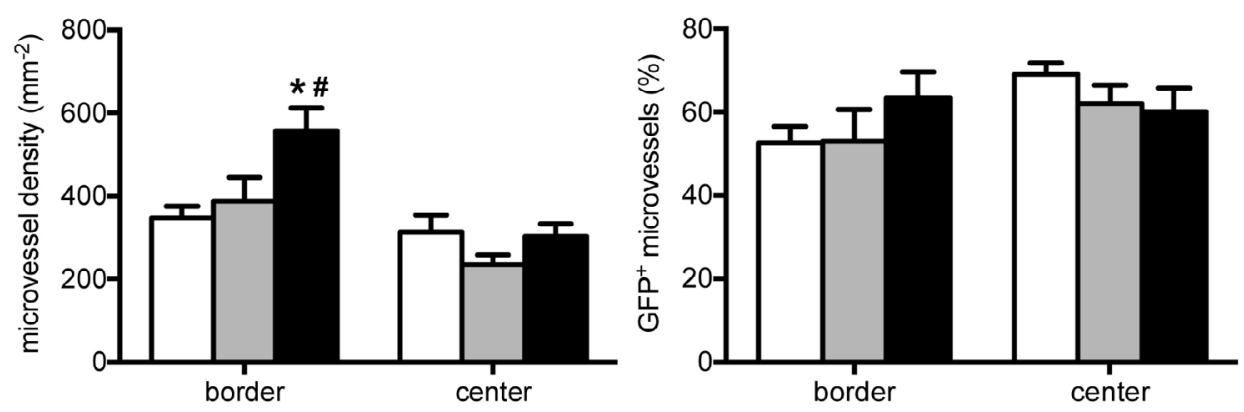

Fig. 5. (a,b) Immunohistochemical detection of $\mathrm{CD} 31^{+}$microvessels (arrows) in the border zones of collagen-glycosaminoglycan matrices (borders marked by dotted lines) seeded with (a) vehicle-treated and (b) EPO-treated ad-MVF on day 14 after implantation into the dorsal skinfold chamber of C57BL/6 mice. The histological sections were stained with Hoechst 33342 to identify cell nuclei (blue) and an antibody against CD31 for the detection of endothelial cells (red). Scale bars: $220 \mu \mathrm{m}$. (c-k) Immunohistochemical characterisation of microvessels in the border zones of collagen-glycosaminoglycan matrices seeded with (f-h) vehicle-treated, (i-k) EPO-treated and (c-e) freshly isolated ad-MVF. The histological sections were stained with Hoechst 33342 to identify cell nuclei (c-k, blue), an antibody against CD31 for the detection of endothelial cells (c,f,i; red) and an antibody against GFP (d, $\mathbf{g}, \mathbf{j}$; green). $\mathbf{e}, \mathbf{h}$ and $\mathbf{k}$ display merges of $(\mathbf{c}, \mathbf{d}),(\mathbf{f}, \mathbf{g})$ and $(\mathbf{i}, \mathbf{j})$, respectively. Arrows $=\mathrm{GFP}^{+} / \mathrm{CD}^{+} 1^{+}$microvessels; arrowheads $=\mathrm{GFP}^{-} /$ $\mathrm{CD} 1^{+}$microvessels. Scale bars: $15 \mu \mathrm{m}$. Microvessel density $\left(\mathbf{1}, \mathrm{mm}^{-2}\right)$ and $\mathrm{GFP}^{+} / \mathrm{CD}^{2} 1^{+}$microvessels percentage $(\mathbf{m}, \%)$ in the border and centre zones of collagen-glycosaminoglycan matrices seeded with vehicle-treated (grey bars, $n=8$ ), EPO-treated (black bars, $n=8$ ) and freshly isolated ad-MVF (white bars, $n=8$ ), as assessed by immunohistochemical analysis. Means \pm SEM. ${ }^{*} p<0.05$ vs. freshly isolated ad-MVF. ${ }^{\#} p<0.05$ vs. vehicle-treated ad-MVF. 
of proliferating endothelial cells was increased, whereas the number of apoptotic perivascular cells was significantly reduced within EPO-treated ad-MVF. Hence, they contained more activated endothelial cells and viable pericytes than vehicletreated and freshly isolated ad-MVF at the time point of matrix seeding and in vivo implantation. Of note, the dynamic interaction of these cells is important for blood vessel development because perivascular cells secrete numerous factors guiding microvascular differentiation (Tattersall et al., 2016). Moreover, they lead sprouting endothelial cells and bridge the gaps between two sprouting segments (Ponce and Price, 2003; Stapor et al., 2014). The latter observation may also crucially contribute to the reassembly of adMVF into microvascular networks. Finally, pericytes stabilise newly formed microvessels, which is a major prerequisite to prevent rapid vessel regression during microvascular remodelling processes (Caporali et al., 2017).

Anti-apoptotic as well as proliferative effects of $\mathrm{EPO}$ on non-haematopoietic tissues are reported and are crucially dependent on the presence of the EPO receptor (Bahlmann et al., 2003; Warren et al., 2011). This receptor is also found on endothelial cells and perivascular cells, i.e. pericytes and smooth muscle cells (Wang et al., 2011), and its expression is upregulated by hypoxia via mitogen-activated protein kinase (MAPK; Cokic et al., 2014). Freshly isolated ad-MVF lack their own blood perfusion and, thus, suffer from hypoxia and oxidative stress (Grässer et al., 2016). Accordingly, it may be speculated that they particularly benefit from EPO treatment in the initial phase after their isolation. Therefore, freshly isolated ad-MVF were cultivated for $24 \mathrm{~h}$ in EPO-supplemented medium prior to their in vivo application. This short ex situ pre-stimulation phase also bears the advantage that potential side effects of $\mathrm{EPO}$ can be avoided, as they may occur if EPO would be administered systemically to the recipient of an ad-MVF-seeded matrix.

The activation of the EPO receptor and its downstream signalling pathway JAK2/STAT5 are involved in the intracellular regulation of numerous pro-angiogenic cytokines (Witthuhn et al., 1993; Zwezdaryk et al., 2007). Accordingly, Jaquet et al. (2002) report that EPO exerts strong angiogenic effects on human endothelial cells, comparable to those of VEGF. Therefore, it was speculated that EPO-treated ad-MVF exhibited a clear pro-angiogenic phenotype when compared with vehicle-treated ones. However, the proteome profiler array showed only modest differences between the two groups. In contrast, when compared with freshly isolated, non-cultivated controls, a strong up-regulation of several pro- and anti-angiogenic factors was detected in both cultivated vehicle-treated and EPO-treated ad-MVF. This indicated a relevant cultivation-induced activation of ad-MVF. In cultivated vehicle-treated ad-MVF, this activation might have compensated for potential negative effects of the reduced viability of perivascular cells on the in vivo vascularisation capacity. Indeed, the newly developing microvascular networks within collagen-glycosaminoglycan matrices seeded with freshly isolated and vehicle-treated ad-MVF exhibited a comparable microvessel density. In the group of cultivated EPO-treated ad-MVF, the cultivation-induced activation in combination with a maintained viability and enhanced endothelial cell proliferation might be the cause for the improved in vivo performance when compared with the other two groups.

The vascularisation capacity of ad-MVF was analysed in the mouse dorsal skinfold chamber model by means of intravital fluorescence microscopy. In contrast to histological approaches, this technique allows for the repetitive analysis of functional, bloodperfused microvessels, which can be identified by means of the plasma marker FITC-labelled dextran (Laschke and Menger, 2016b). Hence, it also enables the measurement of haemodynamic parameters, such as centreline RBC velocities and wall shear rates of microvessels. Importantly, matrices seeded with EPO-treated ad-MVF presented a significantly higher functional microvessel density between days 6 and 14 when compared with matrices seeded with vehicle-treated ad-MVF and freshly isolated controls. This demonstrated that EPO promoted the early development of interconnections between individual ad-MVF and the vessels of the surrounding host microvasculature, resulting in an improved formation of functional microvascular networks within the implants. These networks also underwent an accelerated maturation and remodelling process, as indicated by smaller diameters as well as higher centreline RBC velocities and wall shear rates of individual microvessels when compared with those in the vehicle-treated group. Because increased shear rates stimulate angiogenesis (Galie et al., 2014), these favourable microhaemodynamic conditions might have further contributed to the rapid development of dense microvascular networks in matrices seeded with EPO-treated ad-MVF.

Finally, the vascularisation of the implanted matrices was analysed on day 14 by means of immunohistochemistry. By this, a comparable density of $\mathrm{CD} 31^{+}$microvessels was detected within the centre of matrices seeded with vehicle- and EPO-treated as well as freshly isolated ad-MVF. Moreover, the matrices of all three groups contained a large fraction of $60-70 \% \mathrm{GFP}^{+} / \mathrm{CD}^{+} 1^{+}$microvessels originating from the seeded ad-MVF. Of interest, a significantly increased number of $\mathrm{CD} 31^{+}$microvessels was found in the border tissue surrounding the matrices seeded with EPO-treated ad-MVF when compared with the other two groups, whereas the fraction of $\mathrm{GFP}^{+} /$ CD31 ${ }^{+}$microvessels was comparable. This indicated that i) more $\mathrm{GFP}^{+} / \mathrm{CD} 31^{+}$microvessels grew out of the matrices seeded with EPO-treated ad-MVF and 
ii) more $\mathrm{GFP}^{-} / \mathrm{CD} 31^{+}$microvessels developed in the border zones of these matrices. Based on the in vitro data, it might be assumed that this was the result of an increased endothelial cell proliferation within EPO-treated ad-MVF and their enhanced secretion of pro-angiogenic factors. This might have stimulated the growth of $\mathrm{GFP}^{+} / \mathrm{CD} 31^{+}$microvessels from the implants towards the $\mathrm{GFP}^{-} / \mathrm{CD} 31^{+}$microvessels of the host tissue and vice versa, thus promoting the early interconnection of the two microvascular networks at the border zones.

\section{Conclusions}

The present study demonstrated that EPO maintained the viability and increased the proliferating activity of short-term cultivated ad-MVF. Moreover, treatment with this cytokine stimulated the in vivo reassembly of ad-MVF into microvascular networks after implantation. Hence, the herein introduced approach offered the exciting opportunity to further boost the remarkable vascularisation properties of ad-MVF in future tissue-engineering applications.

\section{Acknowledgements}

We are grateful for the excellent technical assistance of Janine Becker and Julia Parakenings. This study was funded by a grant of the Deutsche Forschungsgemeinschaft (DFG - German Research Foundation) - LA 2682/7-1.

\section{References}

Alural B, Duran GA, Tufekci KU, Allmer J, Onkal Z, Tunali D, Genc K, Genc S (2014) EPO mediates neurotrophic, neuroprotective, anti-oxidant, and anti-apoptotic effects via downregulation of miR-451 and miR-885-5p in SH-SY5Y neuron-like cells. Front Immunol 5: 475.

Bahlmann FH, DeGroot K, Duckert T, Niemczyk E, Bahlmann E, Boehm SM, Haller H, Fliser D (2003) Endothelial progenitor cell proliferation and differentiation is regulated by erythropoietin. Kidney Int 64: 1648-1652.

Calvillo L, Latini R, Kajstura J, Leri A, Anversa P, Ghezzi P, Salio M, Cerami A, Brines M (2003) Recombinant human erythropoietin protects the myocardium from ischemia-reperfusion injury and promotes beneficial remodeling. Proc Natl Acad Sci U S A 100: 4802-4806.

Caporali A, Martello A, Miscianinov V, Maselli D, Vono R, Spinetti G (2017) Contribution of pericyte paracrine regulation of the endothelium to angiogenesis. Pharmacol Ther 171: 56-64.
Cokic BB, Cokic VP, Suresh S, Wirt S, Noguchi CT (2014) Nitric oxide and hypoxia stimulate erythropoietin receptor via MAPK kinase in endothelial cells. Microvasc Res 92: 34-40.

Fischer HS, Reibel NJ, Bührer C, Dame C (2017) Prophylactic early erythropoietin for neuroprotection in preterm infants: a meta-analysis. Pediatrics 139: e20164317.

Frueh FS, Später T, Lindenblatt N, Calcagni M, Giovanoli P, Scheuer C, Menger MD, Laschke MW (2017a) Adipose tissue-derived microvascular fragments improve vascularization, lymphangiogenesis, and integration of dermal skin substitutes. J Invest Dermatol 137: 217-227.

Frueh FS, SpäterT, Scheuer C, Menger MD, Laschke MW (2017b) Isolation of murine adipose tissuederived microvascular fragments as vascularization units for tissue engineering. J Vis Exp: 122.

Galie PA, Nguyen DH, Choi CK, Cohen DM, Janmey PA, Chen CS (2014) Fluid shear stress threshold regulates angiogenic sprouting. Proc Natl Acad Sci U S A 111: 7968-7973.

Grässer C, Scheuer C, Parakenings J, Tschernig T, Eglin D, Menger MD, Laschke MW (2016) Effects of macrophage-activating lipopeptide-2 (MALP-2) on the vascularisation of implanted polyurethane scaffolds seeded with microvascular fragments. Eur Cell Mater 32: 74-86.

Hiscox AM, Stone AL, Limesand S, Hoying JB, Williams SK (2008) An islet-stabilizing implant constructed using a preformed vasculature. Tissue Eng Part A 14: 433-440.

Hoying JB, Boswell CA, Williams SK (1996) Angiogenic potential of microvessel fragments established in three-dimensional collagen gels. In Vitro Cell Dev Biol Anim 32: 409-419.

Jaquet K, Krause K, Tawakol-Khodai M, Geidel S, Kuck KH (2002) Erythropoietin and VEGF exhibit equal angiogenic potential. Microvasc Res 64: 326333.

Kimáková P, Solár P, Solárová Z, Komel R, Debeljak N (2017) Erythropoietin and its angiogenic activity. Int J Mol Sci 18: E1519.

Laschke MW, Harder Y, Amon M, Martin I, Farhadi J, Ring A, Torio-Padron N, Schramm R, Rücker M, Junker D, Häufel JM, Carvalho C, Heberer M, Germann G, Vollmar B, Menger MD (2006) Angiogenesis in tissue engineering: breathing life into constructed tissue substitutes. Tissue Eng 12: 2093-2104.

Laschke MW, Vollmar B, Menger MD (2009) Inosculation: connecting the life-sustaining pipelines. Tissue Eng Part B Rev 15: 455-465.

Laschke MW, Vollmar B, Menger MD (2011) The dorsal skinfold chamber: window into the dynamic interaction of biomaterials with their surrounding host tissue. Eur Cell Mater 22: 147-164.

Laschke MW, Kleer S, Scheuer C, Schuler S, Garcia P, Eglin D, Alini M, Menger MD (2012) Vascularisation of porous scaffolds is improved by incorporation of 
adipose tissue-derived microvascular fragments. Eur Cell Mater 24: 266-277.

Laschke MW, Kleer S, Scheuer C, Eglin D, Alini M, Menger MD (2015) Pre-cultivation of adipose tissuederived microvascular fragments in porous scaffolds does not improve their in vivo vascularisation potential. Eur Cell Mater 29: 190-200.

Laschke MW, Menger MD (2015) Adipose tissue-derived microvascular fragments: natural vascularization units for regenerative medicine. Trends Biotechnol 33: 442-448.

Laschke MW, Menger MD (2016a) Prevascularization in tissue engineering: current concepts and future directions. Biotechnol Adv 34: 112-121.

Laschke MW, Menger MD (2016b) The dorsal skinfold chamber: a versatile tool for preclinical research in tissue engineering and regenerative medicine. Eur Cell Mater 32: 202-215.

McDaniel JS, Pilia M, Ward CL, Pollot BE, Rathbone CR (2014) Characterization and multilineage potential of cells derived from isolated microvascular fragments. J Surg Res 192: 214-222.

Muscari C, Giordano E, Bonafè F, Govoni M, Guarnieri C (2014) Strategies affording prevascularized cell-based constructs for myocardial tissue engineering. Stem Cells Int 2014: 434169.

Nakano M, Satoh K, Fukumoto Y, Ito Y, Kagaya Y, Ishii N, Sugamura K, Shimokawah (2007) Important role of erythropoietin receptor to promote VEGF expression and angiogenesis in peripheral ischemia in mice. Circ Res 100: 662-669.

Novosel EC, Kleinhans C, Kluger PJ (2011) Vascularization is the key challenge in tissue engineering. Adv Drug Deliv Rev 63: 300-311.

Ponce AM, Price RJ (2003) Angiogenic stimulus determines the positioning of pericytes within capillary sprouts in vivo. Microvasc Res 65: 45-48.

Rainville N, Jachimowicz E, Wojchowski DM (2016) Targeting EPO and EPO receptor pathways in anemia and dysregulated erythropoiesis. Expert Opin Ther Targets 20: 287-301.

Shepherd BR, Hoying JB, Williams SK (2007) Microvascular transplantation after acute myocardial infarction. Tissue Eng 13: 2871-2879.

Socolovsky M, Nam H, Fleming MD, Haase VH, Brugnara C, Lodish HF (2001) Ineffective erythropoiesis in Stat $5 \mathrm{a}(-/-) 5 \mathrm{~b}(-/-)$ mice due to decreased survival of early erythroblasts. Blood 98 : 3261-3273.

Stapor PC, Sweat RS, Dashti DC, Betancourt AM, Murfee WL (2014) Pericyte dynamics during angiogenesis: new insights from new identities. J Vasc Res 51: 163-174.

Tattersall IW, Du J, Cong Z, Cho BS, Klein AM, Dieck CL, Chaudhri RA, Cuervo H, Herts JH, Kitajewski J (2016) In vitro modeling of endothelial interaction with macrophages and pericytes demonstrates Notch signaling function in the vascular microenvironment. Angiogenesis 19: 201-215.
Wang L, Chopp M, Gregg SR, Zhang RL, Teng H, Jiang A, Feng Y, Zhang ZG (2008) Neural progenitor cells treated with EPO induce angiogenesis through the production of VEGF. J Cereb Blood Flow Metab 28: 1361-1368.

Wang Q, Gorbey S, Pfister F, Höger S, DornBeineke A, Krügel K, Berrone E, Wu L, Korff T, Lin J, Busch S, Reichenbach A, Feng Y, Hammes HP (2011) Long-term treatment with suberythropoietic Epo is vaso- and neuroprotective in experimental diabetic retinopathy. Cell Physiol Biochem 27: 769-782.

Warren JS, Zhao Y, Yung R, Desai A (2011) Recombinant human erythropoietin suppresses endothelial cell apoptosis and reduces the ratio of Bax to Bcl-2 proteins in the aortas of apolipoprotein E-deficient mice. J Cardiovasc Pharmacol 57: 424-433.

Watanabe D, Suzuma K, Matsui S, Kurimoto M, Kiryu J, Kita M, Suzuma I, Ohashi H, Ojima T, Murakami T, Kobayashi T, Masuda S, Nagao M, Yoshimura N, Takagih (2005) Erythropoietin as a retinal angiogenic factor in proliferative diabetic retinopathy. N Engl J Med 353: 782-792.

Witthuhn BA, Quelle FW, Silvennoinen O, Yi T, Tang B, Miura O, Ihle JN (1993) JAK2 associates with the erythropoietin receptor and is tyrosine phosphorylated and activated following stimulation with erythropoietin. Cell 74: 227-236.

Zwezdaryk KJ, Coffelt SB, Figueroa YG, Liu J, Phinney DG, LaMarca HL, Florez L, Morris CB, Hoyle GW, Scandurro AB (2007) Erythropoietin, a hypoxiaregulated factor, elicits a pro-angiogenic program in human mesenchymal stem cells. Exp Hematol 35: 640-652.

\section{Discussion with Reviewers}

Marietta Herrmann: The improvements by EPOtreatment seemed relatively minor in comparison with the freshly isolated ad-MVF. Would the authors still promote this treatment strategy, in particular in light of potential hurdles associated with the preculture process (e.g. ex vivo manipulation, time)?

Authors: We agree with the reviewer that the precultivation of ad-MVF might be associated with legal and logistic disadvantages when compared with the use of freshly isolated ad-MVF in an intra-operative one-step procedure. However, in clinical practice it might not always be possible to retransfer ad-MVF into patients immediately after their isolation due to prolonged or multi-stage surgical interventions. In such cases requiring the short-term storage of ad-MVF, the herein described EPO treatment would be suitable to further boost the remarkable vascularisation properties of ad-MVF.

Daniel Schmauss: Could you imagine using CEPO (carbamylated EPO without haematogenic effects) in higher doses to achieve even better results without adverse effects? 
Authors: In the present study, isolated ad-MVF from donor animals were exclusively exposed to EPO during a $24 \mathrm{~h}$ short-term cultivation period, whereas the recipient animals did not receive a systemic EPO therapy. Therefore, this approach was not expected to induce any EPO-associated adverse effects, such as thrombus formation, in the recipient animals. Nonetheless, the reviewer's suggestion to use carbamylated EPO is an excellent idea. In fact, carbamylated EPO suppresses apoptosis and promotes angiogenesis without stimulating excessive erythropoiesis upon chronic dosing (Leist et al., 2004; Imamura et al., 2008, additional references). This favourable profile of action would offer the promising opportunity to directly transfer freshly isolated ad-MVF without any pre-cultivation steps into recipient animals, where their network-forming capacity would then be stimulated in vivo by systemic administration of carbamylated EPO. Since this type of treatment could also be performed over a longer period, it may even achieve better results than the herein observed effects of ad-MVF short-term exposure to EPO.

\section{Additional References}

Imamura R, Okumi M, Isaka $\mathrm{Y}$, Ichimaru $\mathrm{N}$, Moriyama T, Imai E, Nonomura N, Takahara S, Okuyama A (2008) Carbamylated erythropoietin improves angiogenesis and protects the kidneys from ischemia-reperfusion injury. Cell Transplant 17: 135141.

Leist M, Ghezzi P, Grasso G, Bianchi R, Villa P, Fratelli M, Savino C, Bianchi M, Nielsen J, Gerwien J, Kallunki P, Larsen AK, Helboe L, Christensen S, Pedersen LO, Nielsen M, Torup L, Sager T, Sfacteria A, Erbayraktar S, Erbayraktar Z, Gokmen N, Yilmaz O, Cerami-Hand C, Xie QW, Coleman T, Cerami A, Brines M (2004) Derivatives of erythropoietin that are tissue protective but not erythropoietic. Science 305: 239-242.

Editor's note: The Scientific Editor responsible for this paper was Juerg Gasser. 